\title{
BMJ Open White matter integrity and cognition in Parkinson's disease: a cross-sectional study
}

\author{
Eirik Auning, ${ }^{1,2}$ Veslemøy Krohn Kjærvik, ${ }^{3}$ Per Selnes, ${ }^{3}$ Dag Aarsland, ${ }^{1,4,5}$ \\ Astrid Haram, ${ }^{2,6}$ Atle Bjørnerud, ${ }^{7,8}$ Erik Hessen, ${ }^{3,9}$ Abdolreza Esnaashari, ${ }^{10}$ \\ Tormod Fladby ${ }^{3}$
}

To cite: Auning $E$, Kjærvik VK, Selnes P, et al. White matter integrity and cognition in Parkinson's disease: a cross-sectional study. BMJ Open 2014;4: e003976. doi:10.1136/ bmjopen-2013-003976

- Prepublication history for this paper is available online. To view these files please visit the journal online (http://dx.doi.org/10.1136/ bmjopen-2013-003976).

Received 8 September 2013 Revised 8 November 2013 Accepted 14 November 2013

CrossMark

For numbered affiliations see end of article.

Correspondence to Dr Eirik Auning; eirikauning@hotmail.com

\section{ABSTRACT}

Objective: We used diffusion tensor imaging (DTI) to test the following hypotheses: (1) there is decreased white matter (WM) integrity in non-demented Parkinson's disease (PD), (2) WM integrity is differentially reduced in PD and early Alzheimer's disease (AD) and (3) DTI changes in non-demented PD are specifically associated with cognitive performance.

Methods: This study included 18 non-demented patients with PD, 18 patients with mild cognitive impairment due to incipient $A D$ and 19 healthy elderly normal control (NC) participants in a cross-sectional design. The participants underwent DTI, and tractbased spatial statistics was used to analyse and extract radial diffusivity and fractional anisotropy. Correlations between scores from a battery of neuropsychological tests and DTI were performed in the PD group.

Results: Patients with PD had significant differences in DTI in WM underlying the temporal, parietal and occipital cortex as compared with NC. There were no significant differences between the $P D$ and $A D$ groups in the primary region of interest analyses, but compared with NC there was a tendency for more anterior changes in $A D$ in contrast to more posterior changes in PD. In a secondary whole-brain analysis there were frontoparietal areas with significant differences between $A D$ and $P D$. In patients with PD, there were significant correlations between DTI parameters in WM underlying the prefrontal cortex and executive and visuospatial abilities.

Conclusions: In early, non-demented PD we found reduced WM integrity underlying the temporal, parietal and occipital cortices. In addition, WM integrity changes in prefrontal areas were associated with executive and visuospatial ability. These findings support that DTI may be an important biomarker in early PD, and that WM changes are related to cognitive impairment in $\mathrm{PD}$.

\section{INTRODUCTION}

Cognitive impairment is common in Parkinson's disease (PD) and leads to patient suffering, caregiver burden and health-related costs. ${ }^{1}$

\section{Strengths and limitations of this study}

- Limitations of our study include lack of postmortem diagnosis. However, in the case of predementia Alzheimer's disease (AD), diagnosis was strengthened by use of cerebrospinal fluid biomarkers, and all patients with Parkinson's disease (PD) had a positive DaTSCAN supporting the clinical diagnosis.

- The sample size is relatively low, and thus power issues make it difficult to differentiate between possible AD-specific and PD-specific changes.

- Not all patients with PD had measurable cognitive impairment. Thus, it is possible that more pronounced diffusion tensor imaging changes and stronger associations with cognition would have been detected if more PD patients had mild cognitive impairment.

Most imaging studies exploring brain changes associated with cognitive impairment in PD have focused on grey matter atrophy, whereas the importance of white matter (WM) integrity has been less explored. MR diffusion tensor imaging (DTI) may broaden our understanding of WM affection in PD. ${ }^{2}$ Recently, DTI of anterior and posterior cingulum fibre tracts were found to be more affected in PD dementia (PDD) as compared with controls, and anterior cingulate fibres were more affected in patients with PDD compared with PD without dementia. ${ }^{3}$ In another recent study it was suggested that WM affection is associated with cognitive impairment in PD, and that brain changes progress in a sequential pattern with hypoperfusion preceding WM damage and grey matter atrophy. ${ }^{4}$

We and others have previously reported that DTI changes are closely associated with cognitive impairment in Alzheimer's disease (AD). ${ }^{5}$ To our knowledge, however, analysis of DTI parameters compared with neuropsychological tests in patients with $\mathrm{PD}$ and $\mathrm{AD}$ has not yet 
been published. This is important in order to single out PD-specific mechanisms of cognitive impairment. Possibly, these parameters may also be used as biomarkers for PD-driven or AD-driven cognitive impairment. We therefore studied DTI in patients with PD without dementia and compared them with age-matched patients with predementia $\mathrm{AD}$ and healthy controls. Our objective was to use DTI to test the following hypotheses: (1) there is decreased WM integrity in non-demented PD, (2) WM integrity in PD is differentially affected from that in early $\mathrm{AD}$ and (3) the DTI changes in PD are specifically associated with cognitive performance.

\section{METHODS}

\section{Subjects}

Eighteen patients with PD from a university-hospital-based neurological outpatient clinic were recruited during 2011 and 2012. Twelve patients were included at the time of PD diagnosis and the remaining six were patients diagnosed with PD during 2010 and 2011 and attending regular clinical controls at the outpatient clinic. The inclusion criteria were diagnosis of $\mathrm{PD},{ }^{6}$ disease duration less than 5 years and either normal cognition, subjective cognitive impairment (SCI) or mild cognitive impairment (MCI) (see table 1 and Cognitive assessment and classification section). The exclusion criteria were dementia (see below), somatic (other than PD), psychiatric or other diseases that might have contributed to cognitive impairment (including drug abuse, moderate or severe depression, solvent exposure, anoxic brain damage and active cancer).

We included two comparison groups: 18 patients with MCI due to $\mathrm{AD}^{7}$ from an ongoing large MCI study in the same university-hospital and who also participated in our recent DTI study. ${ }^{5}$ These patients were matched in terms of age, gender and level of education to the PD group. To be included, an abnormal score on at least one cerebrospinal fluid (CSF) AD-marker (either CSF-P- $\tau$ or CSF
$\mathrm{AB} 42)$ as recommended ${ }^{7}$ was required. In addition, all patients had memory problems, either subjectively or reported by relatives. CSF extraction was performed by lumbar puncture and analysed according to protocol and as previously described. ${ }^{8}$ CSF P- $\tau$ was considered pathological if $\geq 80 \mathrm{ng} / \mathrm{L}, \mathrm{A} \beta 42$ if $\leq 550 \mathrm{ng} / \mathrm{L}$ and T- $\tau>$ if $450 \mathrm{ng} /$ $\mathrm{L}$ (age 50-69) or $500 \mathrm{ng} / \mathrm{L}$ (age $\geq 70$ ). ${ }^{9}$ The inclusion criteria were aged 40-79 years and subjectively impaired cognition for at least 6 months. The exclusion criteria were significantly impaired activities of daily living (ie, dementia), established psychiatric disorder, cancer, drug abuse, solvent exposure and anoxic brain damage including stroke. The included patients did not fulfil any of the core or suggestive criteria for dementia with Lewy bodies (DLB) or criteria consistent with a diagnosis of frontotemporal dementia.

Nineteen healthy, elderly and cognitively normal control (NC) participants were selected from spouses or relatives of the patients with AD-MCI on the basis of a clinical interview by a neurologist and neuropsychological tests administered by a trained neuropsychologist (ie, T-scores $\geq 40$ on tests of memory, executive functioning and visuospatial ability). NC was individually matched for age and level of education to the PD and AD-MCI groups.

\section{Clinical assessment}

All patients with PD were examined by a neurologist with training in movement disorders and met the criteria of probable PD (3 of 4 features: asymmetric onset, bradykinesia, rigidity and resting tremor), ${ }^{6}$ with the exception that the requirement of disease duration more than 3 years was not met by 13 patients (see table 1). To further support the PD diagnosis, all participants had a pathological single photon emission CT using an ioflupane $\left({ }^{123} \mathrm{I}\right)$ biomarker (DaTSCAN) ${ }^{10}$

Standardised rating scales of motor function (Unified Parkinson's Disease Rating Scale (UPDRS) motor

Table 1 Demographics and clinical characteristics

\begin{tabular}{|c|c|c|c|c|}
\hline Variables & AD-MCl† $(n=18)$ & $P D \ddagger(n=18)$ & Controls $(n=19)$ & p Valus* \\
\hline Age, years & $65.6 \pm 5.6(55-77)$ & $66.7 \pm 5.1(59-75)$ & $64.6 \pm 6.5(52-77)$ & 0.537 \\
\hline Female sex, $n$ & $9(50 \%)$ & $9(50 \%)$ & $13(68 \%)$ & 0.424 \\
\hline Disease duration, years & $2.4 \pm 1.7(0.5-7)$ & $2.2 \pm 1.1(1-5)$ & - & 0.181 \\
\hline MMSE§ score & $27.3 \pm 1.8(24-30)$ & $28.8 \pm 1.4(26-30)$ & $29.5 \pm 0.5(29-30)$ & 0.000 \\
\hline GDSף, categories $1 / 2 / 3, n$ & $0 / 0 / 18$ & $8 / 3 / 7$ & $19 / 0 / 0$ & 0.000 \\
\hline Education, years & $12.8 \pm 3.3(8-18)$ & $11.3 \pm 3.5(7-18)$ & $11.8 \pm 2.4(8-16)$ & 0.345 \\
\hline Fazekas; mean white matter score & $1.0 \pm 0.8(0-2)$ & $0.9 \pm 0.7(0-2)$ & $0.8 \pm 0.6(0-2)$ & 0.779 \\
\hline Fazekas; mean periventricular score & $1.1 \pm 0.5(0-2)$ & $1.1 \pm 0.5(0-2)$ & $1.1 \pm 0.5(0-2)$ & 0.927 \\
\hline Cerebrovascular composite score ${ }^{* *}$ & $1.7 \pm 1.0(0-3)$ & $1.6 \pm 1.4(0-4)$ & - & 0.894 \\
\hline
\end{tabular}

The three groups did not differ regarding age, sex, years of education, cerebrovascular burden and Fazekas score.

Numbers represent means $\pm \mathrm{SD}$ (range) unless otherwise indicated.

*Significant at the $\mathrm{p}<0.05$ level.

†Alzheimer's disease-mild cognitive impairment.

†Parkinson's disease.

§Mini-Mental State Examination.

ๆGlobal Deterioration Scale, category 1=normal, category 2=subjective cognitive impairment, category 3=mild cognitive impairment.

${ }^{* *}$ Composite score from 0 to 6 were 0 indicates no cerebrovascular burden, 6 indicates a maximum disease burden. 
subscale ${ }^{11}$ and Hoehn and Yahr $^{12}$ staging were performed by trained research physicians. The mean UPDRS motor and Hoehn and Yahr scores were 12.3 \pm 6.8 SD (range 5-31) and 1.7 $\pm 0.6 \mathrm{SD}$ (range 1.0-2.5), respectively. Fifteen patients with PD used dopaminergic medication, mean total daily levodopa equivalent dose $361 \pm 186$ SD (range 100-632). ${ }^{13}$ At inclusion, 10 of 18 patients used levodopa, 5 patients used dopaminagonists and levodopa, 5 patients used only dopaminagonists and 3 patients used no dopaminergic medication. Three patients used antidepressants; no patients used benzodiazepines, antipsychotics or acetylcholinesterase inhibitors.

Depression was assessed using the Geriatric Depression Scale. ${ }^{14}$ No patients with depressive symptoms above $5 / 6$ cut-off were included, and the mean scores were $2.4 \pm 1.3$ and $1.2 \pm 1.0 \mathrm{SD}$ for the $\mathrm{AD}$ and $\mathrm{PD}$ groups, respectively, the $\mathrm{AD}$ group having slightly more depressive symptoms than the PD group ( $\mathrm{p}=0.043)$.

Since DTI changes may also relate to cerebrovascular disease, WM lesions (WMLs) in the PD, $\mathrm{AD}$ and $\mathrm{NC}$ groups were assessed using the method published by Fazekas $e t a l^{15}$ by one reviewer with good test-retest reliability (Pearson correlation coefficient 0.83 and 0.92 for periventricular and subcortical WML, respectively). There were no significant differences between the groups (table 1). In addition a composite cerebrovascular risk score in the three groups was made consisting of six items (smoking, diabetes, hypertension, hypercholesterolaemia (ie, total cholesterol above $7 \mathrm{mmol} / \mathrm{L}$ or use of anticholesterol agents), hyperhomocysteinaemia (ie, above $15 \mu \mathrm{mol} / \mathrm{L}$ ) and known cerebrovascular disease other than stroke and/or known cerebrovascular disease in the family). The total cerebrovascular burden in the individual patient was thus assessed on a scale from 0 to 6 , where 0 indicated no burden and 6 indicated maximum burden of disease. There were no significant differences in the mean cerebrovascular burden among the $\mathrm{AD}, \mathrm{PD}$ and NC groups (table 1).

\section{Cognitive assessment and classification}

All participants performed a cognitive assessment programme, which consisted of three steps. In addition to a clinical interview, Mini-Mental State Examination (MMSE) and domain-specific screening tests were performed by the clinician. As previously described, cognitive staging (in normal, SCI and MCI) according to the Global Deterioration Scale was performed. ${ }^{16}$

In addition, tests of memory, executive functioning and visuospatial ability were administered by a trained neuropsychologist. The domains selected were chosen as typically impaired in PD and/or AD. ${ }^{17}$ The Rey Auditory Verbal Learning Test-Delayed Recall (RAVLT-DR) was used to measure episodic verbal memory. ${ }^{18}$ Visuospatial ability was assessed using the Rey Complex Figure Test (RCFT; reference area $0-36$ points). ${ }^{19}$ In RAVLT-DR and RCFT raw scores were used to analyse correlations to DTI parameters. Aspects of executive functions were assessed by tests measuring divided attention (Trail Making Test-B
(TMT-B) $),{ }^{20}$ response inhibition (Delis-Kaplan Executive Function System (D-KEFS) Color Word, subtest 3) ${ }^{21}$ and word fluency (Controlled Oral Word Association Test, COWAT) ${ }^{22}$ The results for the latter tests were converted into T-scores, a normally distributed scale with a mean score of 50 and an SD of 10. T-scores for TMT-B and COWAT were calculated based on normative data provided by Heaton et al, ${ }^{22}$ and the test of response inhibition was scored according to norms by Delis et al. ${ }^{21} \mathrm{~A}$ composite mean T-score was calculated for the executive tests (equal weighting). These tests were used for exploring associations between cognition and DTI.

\section{Selection of WM region of interest}

Two region of interests (ROIs) for each cognitive domain (memory, executive functioning and visuospatial ability) were selected a priori on the basis of presumed predilection sites for WM tract changes (figure 1). ${ }^{17}$

Memory is associated with entorhinal and parahippocampal cortices, and the WM underlying these ROIs were hence selected regarding this domain.
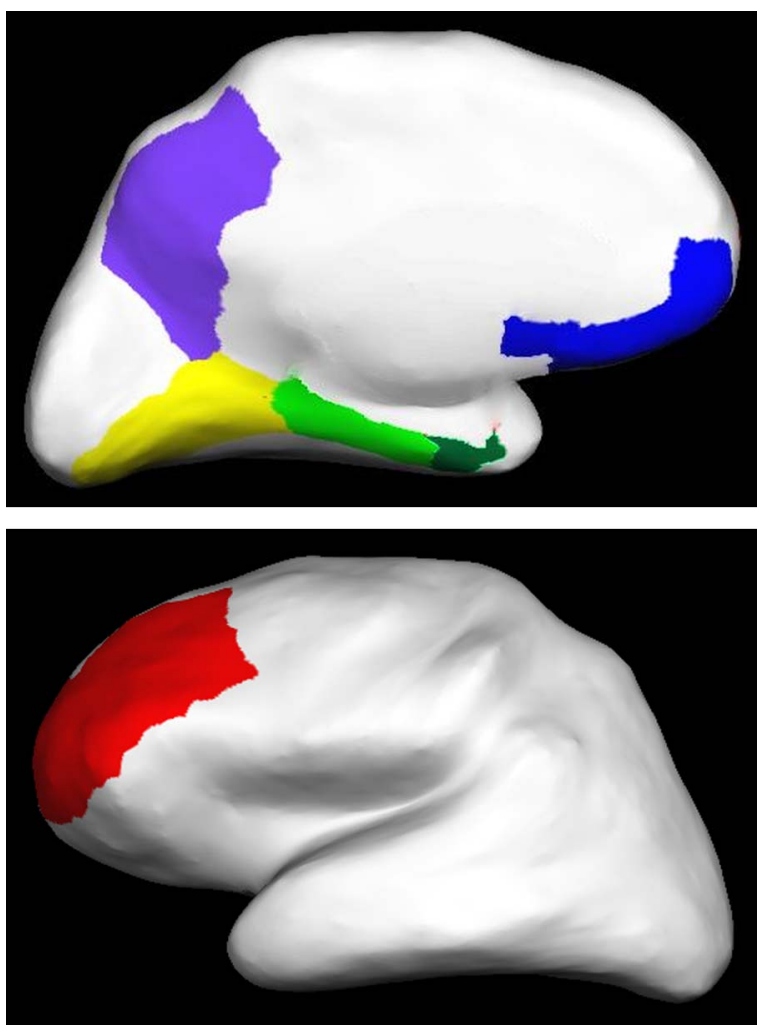

Figure 1 An automated labelling system for subdividing the human cerebral cortex on MRI into gyral-based regions of interest (ROI), by Desikan et al. ${ }^{38}$ Presented are cortical representations of the six preplanned ROls, here shown in one hemisphere. The left picture illustrates the lateral view of the hemisphere, the right showing the medial view of the hemisphere. Red=the middle frontal gyrus (rostral division), blue=the orbitofrontal cortex (medial division), yellow=lingual cortex, light green=parahippocampal cortex, dark green=entorhinal cortex, purple=precuneus. 
Visuoperceptual abnormalities are associated with posterior cortical dysfunction and cause deficits in visual processing in the temporal, parietal and occipital lobe. ${ }^{23-26}$ Occipital hypoperfusion may underlie impairment of visual cognition. ${ }^{27}$ Visual information is processed in two parallel pathways, the ventral occipitotemporal pathway and the dorsal occipitoparietal pathway. ${ }^{29}$ Both extend from the primary visual cortex (V1), but the ventral pathway diverges downwards to visual association areas in the inferior temporal region, and the dorsal pathway diverges upwards to the posterior parietal cortex. Patients with PD are believed to have dysfunction in both. $^{30}$ The neuroanatomical substrate of visuospatial/ visuoperceptual abilities thus involves different cerebral regions, but the occipital lobe is considered to be the most task-specific region concerning visual processing. Based on the presumed anatomical basis of visuospatial function, we hence selected one WM ROI underlying the occipital cortex, that is, the lingual region (adjacent to the parahippocampal cortex) and one WM ROI underlying the posterior parietal cortex, that is, the precuneus region (adjacent to the lingual cortex).

Although dependent on widely distributed networks, neuroimaging studies suggest that executive functions are closely connected to the prefrontal regions of the frontal lobe ${ }^{31}$ further subdivided into three frontosubcortical circuits, namely the dorsolateral prefrontal cortex (DLPFC; eg, verbal fluency, reasoning and working memory), the orbitofrontal cortex (ie, inhibition) and the anterior cingulate cortex (ie, response conflict and emotional drives). ${ }^{32}$ According to a narrow definition, the DLPFC is roughly equivalent to Brodmann area 9 and 46, that is, the middle frontal lobe. We therefore selected the WM underlying the middle frontal gyrus (rostral division) and the WM underlying the orbitofrontal cortex (medial division) for further analysis.

Measurements were averaged between hemispheres.

\section{MRI/DTI acquisition}

MRI was performed at $1.5 \mathrm{~T}$ using a Siemens Espree system (Siemens Healthcare, Erlangen, Germany). Three-dimensional T1-weighted images were acquired using a magnetisation-prepared rapid acquisition gradient echo (MPRAGE) sequence (TR/TE/TI/FA=2400/3.65/ $1000 / 8^{\circ}, \quad$ matrix $\left.=240 \times 192\right), \quad 160 \quad$ sagittal slices, thickness $=1.2 \mathrm{~mm}$, in-plane resolution of $1 \mathrm{~mm} \times 1.2 \mathrm{~mm}$. The protocol also included two-dimensional axial fluid-attenuated inversion recovery images with the following parameters: TR/TE/TI $=13420 / 121 / 2500,36$ slices, spaced at $3 \mathrm{~mm}$ and $3.9 \mathrm{~mm}$ thick.

DTI was acquired using a single shot echo planar imaging sequence with the following parameters: $\mathrm{b}=750 \mathrm{~s} / \mathrm{mm}^{2} ; 12$ diffusion directions repeated five times; 5 b0-values per slice, TR=6100 ms, TE $=117 \mathrm{~ms}$, number of slices: 30 , slice thickness: $3 \mathrm{~mm}$ (gap $1.9 \mathrm{~mm}$ ), in-plane resolution: $1.2 \times 1.2 \mathrm{~mm}^{2}$, bandwidth: $840 \mathrm{~Hz} /$ pixel.

\section{MRI segmentations and analyses}

The Oxford Centre for Functional MRI of the Brain (FMRIB) Software Library (FSL) V.4.1 ${ }^{33}$ was used for DTI analyses and calculations. Initially, FMRIB's Linear Image Registration $\mathrm{Tool}^{23}$ was used for affine registrations of each DTI volume to the low- $b(b=0)$ image.

Fractional anisotropy (FA) and eigenvalue maps were created. Radial diffusivity (DR) is defined as the mean of eigenvalue 2 and 3. Tract-Based Spatial Statistics ${ }^{34}$ was used for voxel-wise statistical analysis of the DTI variables (FA and DR). FMRIB's Diffusion Toolbox was used to create DTI by fitting a tensor model to the raw diffusion data, and FSL's Brain Extraction Tool was used for subsequent brain extraction. All participants' FA data were then aligned into a common space using a non-linear registration tool (FMRIB) which uses a B-spline representation of the registration warp field. ${ }^{35}$ Further, the mean FA image was created and thinned to create a mean FA skeleton that represents the centres of all tracts common to the group. Each participant's aligned FA data were then projected onto this skeleton and the resulting data fed into voxel-wise cross-subject statistics. DR data were then extracted from each participant according to the skeletonised FA map. Moreover, WM ROIs based on the FreeSurfer WM parcellations were extracted for FA and DR-the FSL FMRIB FA template (to which every participant's FA volume initially was registered) was co-registered to the standard space $\mathrm{T} 1$ volume MNI152, which subsequently went through the FreeSurfer processing stream to create a volume with WM parcellations. The processing stream includes segmentation of the subcortical WM and deep grey matter volumetric structures ${ }^{36}$ and parcellation of the cortical surface $^{37}$ according to a previously published parcellation scheme. ${ }^{38}$ This labels cortical sulci and gyri, and thickness values are calculated in the ROIs. Based on the cortical parcellation, WM in the gyrus underneath each cortical label was identified. Each WM voxel within a gyrus was labelled according to the label of the nearest cortical voxel. Deep WM was not assigned to a particular cortical area, with a $5 \mathrm{~mm}$ distance limit. The registration between the FA template and the MNI152 volume was applied to the volume with the WM parcellations, and the resulting volume was used to extract the skeletonised DR data from each WM ROI.

\section{Statistics}

SPSS/PASW statistics V.18 was used for statistical analyses and $\mathrm{p} \leq 0.05$ was considered statistically significant. Comparisons among the three groups ( $\mathrm{PD}, \mathrm{AD}$ and $\mathrm{NC}$ ) were made using one-way analysis of variance (ANOVA), Kruskal-Wallis test, Student t test, Mann-Whitney test or $\chi^{2}$ test as appropriate (table 1 ). The DTI WM ROI variables were normally distributed (after the effects of age and sex had been corrected by regression analysis), and hence one-way ANOVA (with a priori planned contrast $\mathrm{AD}$ vs $\mathrm{NC}$ and $\mathrm{PD}$ vs $\mathrm{NC}$ including polynomial linear trends) was used to compare the three groups. 
Unbiased, voxel-based whole-brain analysis was also performed. However, due to the small sample sizes resulting in low statistical power to detect expected small differences between $\mathrm{AD}$ and $\mathrm{PD}, \mathrm{ROI}$ analyses were chosen as the primary analyses.

The relationship between cognition and DTI in PD was assessed as follows:

Primarily, Pearson correlations between each of the three cognitive domains with the two a priori selected brain regions were determined. In secondary analyses, we determined Pearson correlations between all six ROIs and the three cognitive domains. Finally, the specific executive tests were correlated with the two frontal regions.

The effects of sex and age on the DTI DR parameters were corrected by linear regression before correlations were determined.

\section{RESULTS}

Demographics and clinical characteristics (age, gender, disease duration, education and Fazekas score) did not differ among the groups (table 1). The mean MMSE score was lower in the $\mathrm{AD}$ and $\mathrm{PD}$ groups than $\mathrm{NC}(\mathrm{p}<0.001)$, and the MMSE scores in $\mathrm{AD}(27.3 \pm 1.8 \mathrm{SD})$ were slightly lower than that in PD $(28.8 \pm 1.4 \mathrm{SD} ; \mathrm{p}=0.009)$. The locations of the selected ROI's are illustrated in figure 1.

\section{DTI changes in PD compared with AD and NC subjects}

The ANOVA-analysis revealed significant between-group differences in all preplanned DTI ROIs.

In (temporal ROIs) WM underlying the entorhinal cortex (ERC-WM), DR was higher compared with NC in AD $(p=0.002)$, but did not reach significance in PD ( $\mathrm{p}=0.059$; figure 2). DR in ERC-WM increased from $\mathrm{NC}$ to $\mathrm{PD}$ to $\mathrm{AD}$ (linear trend $\mathrm{p}=0.002$ ). Similar findings emerged for $\mathrm{WM}$ underlying the parahippocampal cortex, that is, AD $(\mathrm{p}<0.001)$ and $\mathrm{PD}(\mathrm{p}=0.030)$ as compared with $\mathrm{NC}$, with a gradient $\mathrm{NC}<\mathrm{PD}<\mathrm{AD}$ (linear trend $\mathrm{p}<0.001)$.

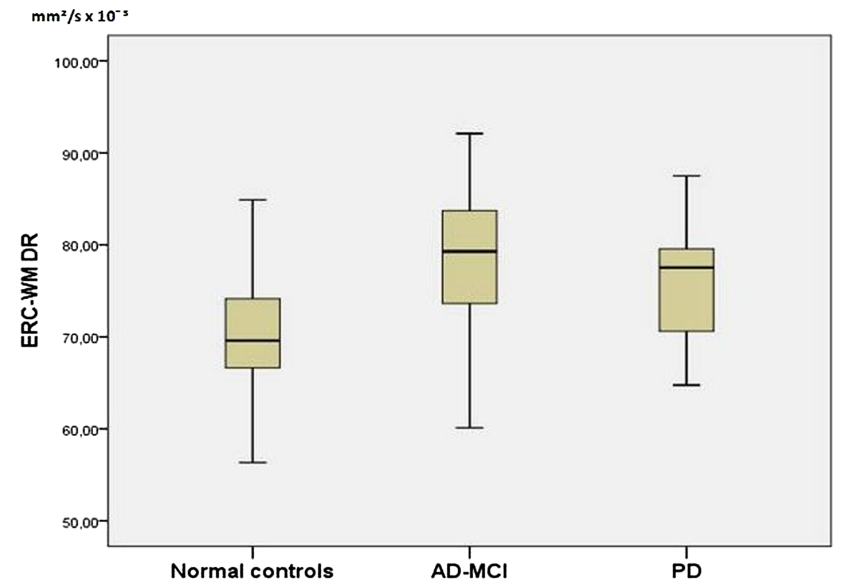

Figure 2 Radial diffusivity in white matter underlying entorhinal cortex (ERC-WM) in Alzheimer's disease mild cognitive impairment (AD-MCI), Parkinson's disease (PD) and normal controls.

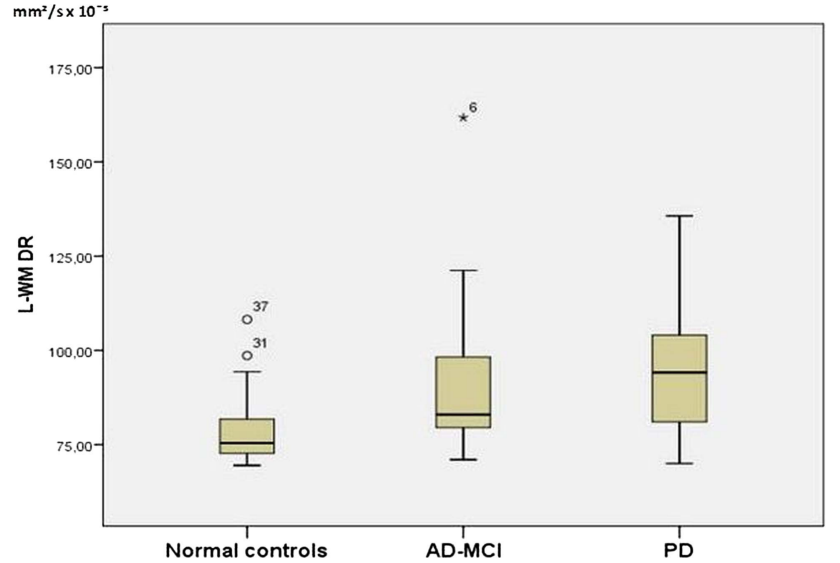

Figure 3 Radial diffusivity in white matter underlying the lingual gyrus (L-WM DR) in Alzheimer's disease mild cognitive impairment (AD-MCl), Parkinson's disease (PD) and normal controls.

In the occipital ROI, higher DR (as compared with NC) was found in WM underlying the lingual gyrus in PD ( $\mathrm{p}=0.034)$, but not in $\mathrm{AD}(\mathrm{p}=0.071)$ with a gradient $\mathrm{NC}<\mathrm{AD}<\mathrm{PD}$ (linear trend $\mathrm{p}=0.034$ ); figure 3 ).

In the parietal ROI (WM underlying the precuneus cortex), higher DR (as compared with $\mathrm{NC}$ ) was found in PD $(\mathrm{p}=0.020)$ and $\mathrm{AD}(\mathrm{p}=0.003)$, with a gradient $\mathrm{NC}<\mathrm{AD}<\mathrm{PD}$ (linear trend $\mathrm{p}=0.007$ ).

The AD-MCI and PD group did not differ regarding the disease duration.

In (frontal ROIs) WM underlying the rostral middle frontal cortex (RMFC-WM) had higher $\mathrm{DR}$ in $\mathrm{AD}$ ( $\mathrm{p}=0.019)$, but not in PD $(\mathrm{p}=0.213)$ as compared with $\mathrm{NC}$ (gradient $\mathrm{NC}<\mathrm{PD}<\mathrm{AD}$ ).

Similar findings were found for WM underlying the medial orbitofrontal cortex, that is, higher $\mathrm{DR}$ in $\mathrm{AD}$ $(p=0.005)$, but not in PD $(p=0.102)$, with a gradient $\mathrm{NC}<\mathrm{PD}<\mathrm{AD}$ (linear trend $\mathrm{p}=0.020$ ).

There were no significant differences in DR in any of the WM ROIs between the PD and AD groups. In a secondary analysis we found similar between group differences for FA (data not shown).

The voxel-based analyses were generally consistent with the findings from the ROI analyses.

In addition to the expected findings between $\mathrm{AD}$ versus NC (DR; figure 4) and PD versus NC (FA; figure 5), there were significant differences in DTI parameters (DR) between $\mathrm{AD}$ and $\mathrm{PD}$, with higher $\mathrm{DR}$ in $\mathrm{AD}$ compared with PD in frontoparietal regions on the left side (corrected for multiple comparisons using threshold-free cluster enhancement; figure 6). However, no significant changes were found for $\mathrm{FA}$ in $\mathrm{AD}$ versus $\mathrm{NC}$ or $\mathrm{AD}$ versus PD, and similarly for DR between PD versus NC.

\section{Associations between DTI and cognition in PD}

T-scores for the different neuropsychological tests in the PD group showed the following (mean and SD): TMT-B $(36.7 \pm 21.1)$, response inhibition $(46.6 \pm 14.3)$, word 


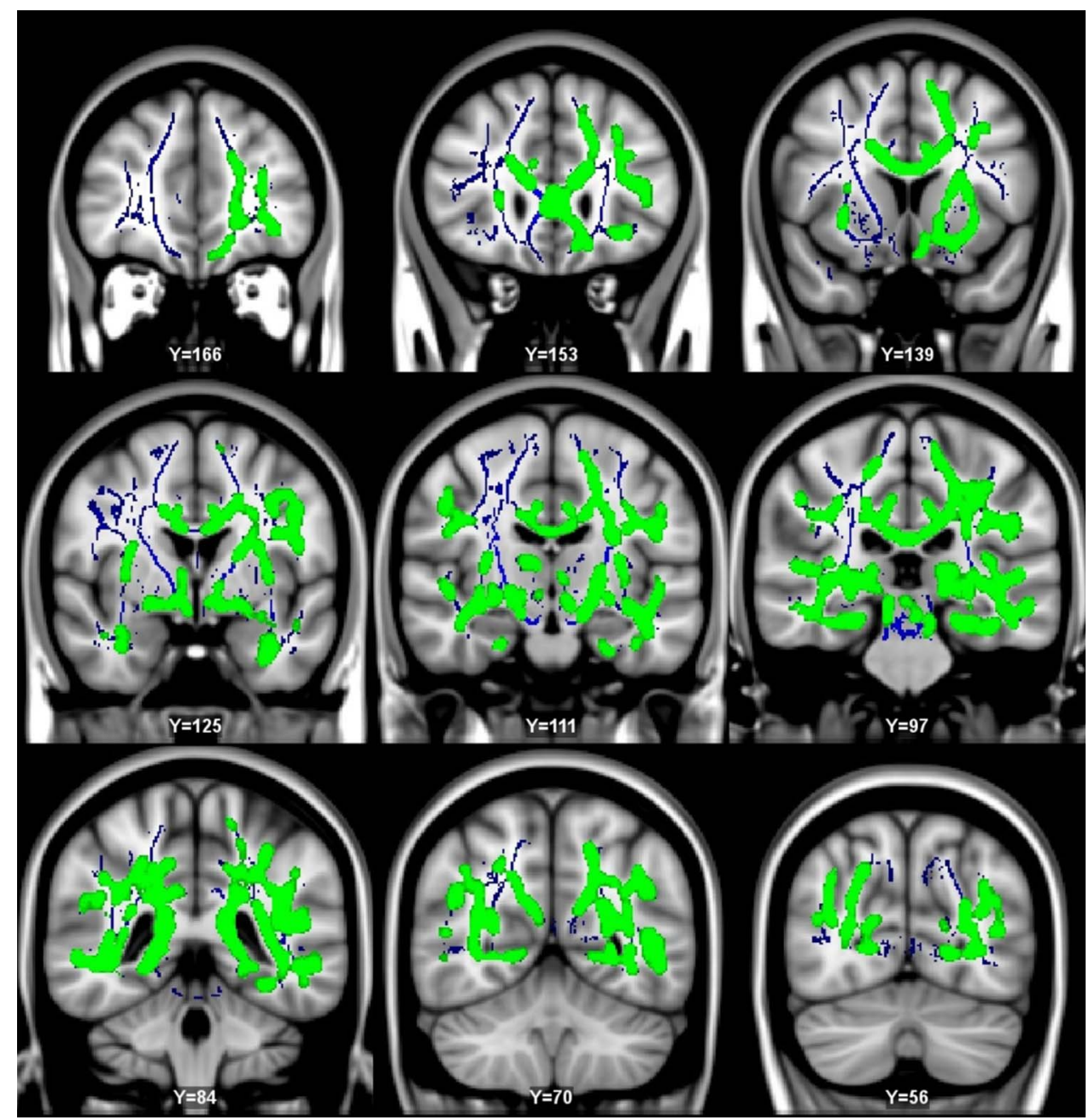

Figure 4 Regions of increased radial diffusivity (DR; green) in patients with Alzheimer's disease as compared with control participants superimposed on the mean fractional anisotropy map from all participants (blue). Widespread differences in both hemispheres are seen. No voxels were significant for reduced DR. Multiple comparisons were corrected for by threshold-free cluster enhancement with the threshold set at $p<0.05$, and the significant voxels are inflated for ease of viewing. The statistical maps are shown as overlays on the Montreal Neurological Institutes template (annotated with the corresponding y-coordinates).

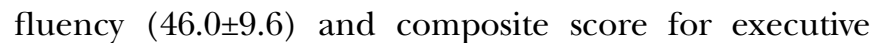
tests $(43.1 \pm 12.0)$. The mean and SD for raw scores on RAVLT and RCFT were 7.1 \pm 3.2 and $28.5 \pm 9.2$, respectively.

No correlations were found between memory (RAVLT-DR) and prespecified DTI variables. Complex figure reproduction did not correlate with the occipital ROI, but in the secondary analyses a correlation with DR in the RMFC-WM was found $(\mathrm{r}=-0.57$; $\mathrm{p}=0.014)$.

When using the composite score for executive functions, we found a significant correlation to DR in RMFC-WM ( $\mathrm{r}=-0.49 ; \mathrm{p}=0.041)$. Analysing subtests of executive functioning (raw scores), we found a correlation between DR in RMFC-WM and time to complete the color-word interference test $(\mathrm{r}=0.51 ; \mathrm{p}=0.030)$ and TMT-B ( $r=0.55: \mathrm{p}=0.019)$.

Voxel-based whole-brain analyses revealed no significant correlations between DTI parameters (DR and FA), memory and composite score for executive functions. Similarly, there were no correlations between DR and performance on RCFT. We found, however, significant correlations between FA and visuoconstruction (RCFT) in corpus callosum (figure 7).

\section{DISCUSSION}

The main finding in this study was that patients with PD without dementia had significant differences in WM DTI-derived metrics in WM underlying the temporal, parietal and occipital cortex as compared with NC. Thus, our findings support the first hypothesis, that WM integrity is disturbed even in early PD.

Our second hypothesis was that there would be differences in DTI parameters between AD and PD. Although we did not find significant differences between the two groups in the primary ROI-based analysis, patients with PD and AD differed from NC in the hypothesised direction (DR in the temporal ROIs $\mathrm{NC}<\mathrm{PD}<\mathrm{AD}$ and in the parietal and occipital ROIs $\mathrm{NC}<\mathrm{AD}<\mathrm{PD}$ ). In voxel-wise whole-brain analysis we 


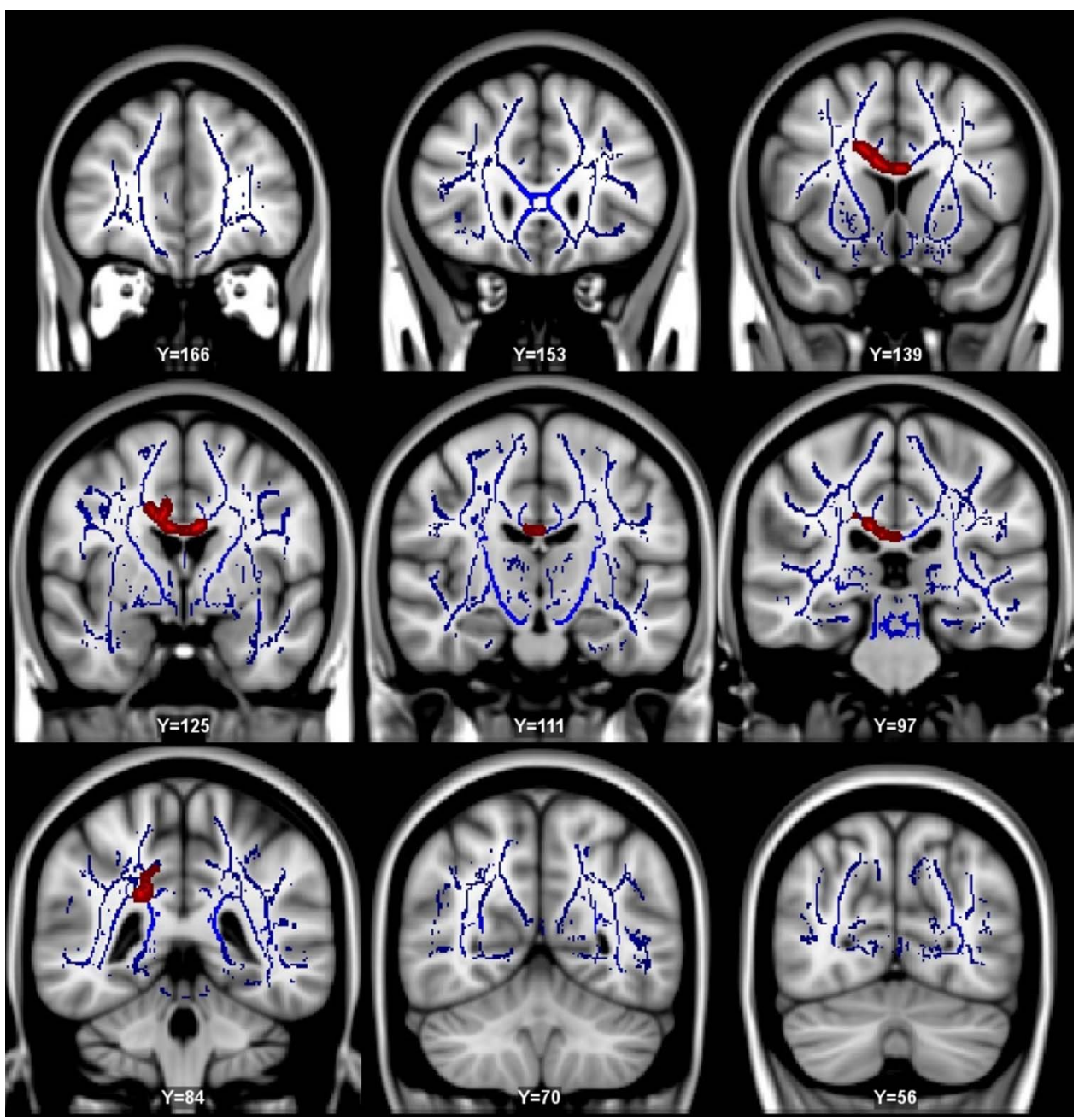

Figure 5 Regions of decreased fractional anisotropy (FA; red) in patients with Parkinson's disease compared with control participants superimposed on the mean FA map from all participants (blue). Significant changes are mainly seen in frontoparietal regions, corpus callosum and the posterior parts of cingulum. No voxels were significant for increased FA. Multiple comparisons were corrected for by threshold-free cluster enhancement with the threshold set at $p<0.05$, and the significant voxels are inflated for ease of viewing. The statistical maps are shown as overlays on the Montreal Neurological Institutes template (annotated with the corresponding y-coordinates).

found, however, significant changes between $\mathrm{AD}$ and PD (DR) in mainly frontoparietal regions including parts of the corticospinal tracts, that is, higher $\mathrm{DR}$ for $\mathrm{AD}$. In addition, the voxel-wise whole-brain analyses showed significant DTI associations in corpus callosum with visuoconstruction.

Our final hypothesis, that DTI changes in PD are associated with cognitive ability, was also at least partly supported. Although we did not find any significant correlations between performance on memory tasks and DTI DR, there were moderately strong correlations between DR in WM underlying the prefrontal cortex and executive tests as well as visuospatial ability.

Our results are consistent with the recent findings that patients with PD, PDD and DLB have more pronounced WM affection than NC. ${ }^{3} 4{ }^{39-41}$ Patients with PD with significant DTI changes in these studies had, however, more severe cognitive impairment (lower mean MMSE scores) than in our PD group. Of note, Hattori et al found no significant DTI changes in the
PD cognitively normal group as compared with NC. In contrast we found significant WM tract changes in a mixed group of patients with $\mathrm{PD}$ with and without MCI. However, in a recent study by Meltzer et $a t^{42}$ mean diffusivity differences (but not FA) were also found in PD cognitively normal participants (relative to $\mathrm{NC}$ ) confined to corpus callosum, but not in other brain regions. Despite a cross-sectional design, the same study also suggested that microstructural WM integrity loss increases with cognitive deterioration. In another recent study, WM abnormalities were not found in PD cognitively normal participants, in contrast to a distributed pattern of WM changes (reduced FA), mainly in frontal areas, in patients with PD with MCI. ${ }^{43}$ Taken together, these findings lend support to the idea that WM tract changes evolve in parallel to cognitive decline in PD.

We did not find correlations between loss of (presumably) visual-tract WM integrity in parieto-occipital 


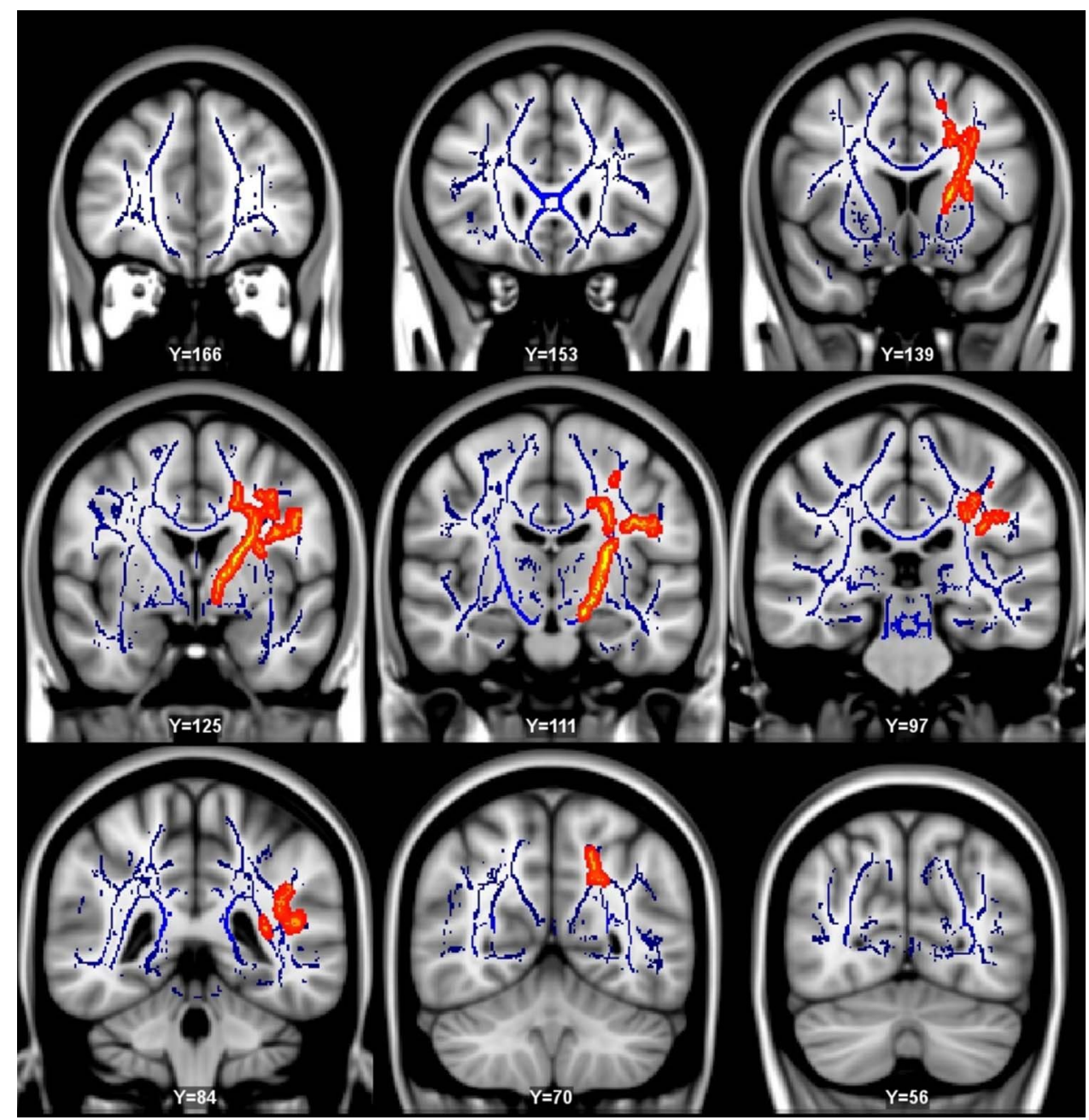

Figure 6 Regions of increased radial diffusivity (DR; red) in patients with Parkinson's disease compared with Alzheimer's disease superimposed on the mean fractional anisotropy map from all participants (blue). Significant changes are seen mainly in frontoparietal regions on the left side including parts of the corticospinal tracts. No voxels were significant for reduced DR. Multiple comparisons were corrected for by threshold-free cluster enhancement with the threshold set at $p<0.05$, and the significant voxels are inflated for ease of viewing. The statistical maps are shown as overlays on the Montreal Neurological Institutes template (annotated with the corresponding y-coordinates). All significant changes shown reflect higher DR in Alzheimer's disease.

regions and tests of visuoconstruction. In the study by Watson et $a t^{40}$ the widespread correlations found between executive functions and parameters of DTI in DLB could be explained by the more advanced disease stage or the potentially different disease progression in DLB and PD. Our study found associations between prefrontal DTI changes and executive functioning in a similar pattern as described by Meltzer et al. ${ }^{42}$ Although there were numerical differences in DTI parameters in prefrontal cortex between PD and NC, they were not statistically significant. This may be due to the small sample size, and stronger associations might have been found if other prefrontal ROIs had been selected. In addition, the complexity of neuronal networks and different brain areas involved in neuropsychological testing make it difficult to exactly localise neuroanatomical substrates of disease. The few DTI studies available in Lewy body disease also suggest that WM changes are rather widespread even at an early disease stage.

Of note, in our study $\mathrm{AD}$ and $\mathrm{PD}$ cases had different mean MMSE scores, which might have influenced the findings. This is not surprising since per definition all patients with $\mathrm{AD}$ had MCI, whereas some of the patients with PD were cognitively intact, and the MMSE emphasises language and memory items and thus is more sensitive to the changes in early AD than in PD. Although significant, the differences in depression scores between patients with $\mathrm{AD}$ and PD were minor and below cut-off for possible depression, and we do not think this had any major influence on the results. Yet, we cannot entirely exclude the possibility that minor differences in overall cognition affected the comparison between $\mathrm{AD}$ and PD.

However, regardless of the complexity of neuronal disease, the clinical correlates to DTI changes in Lewy 


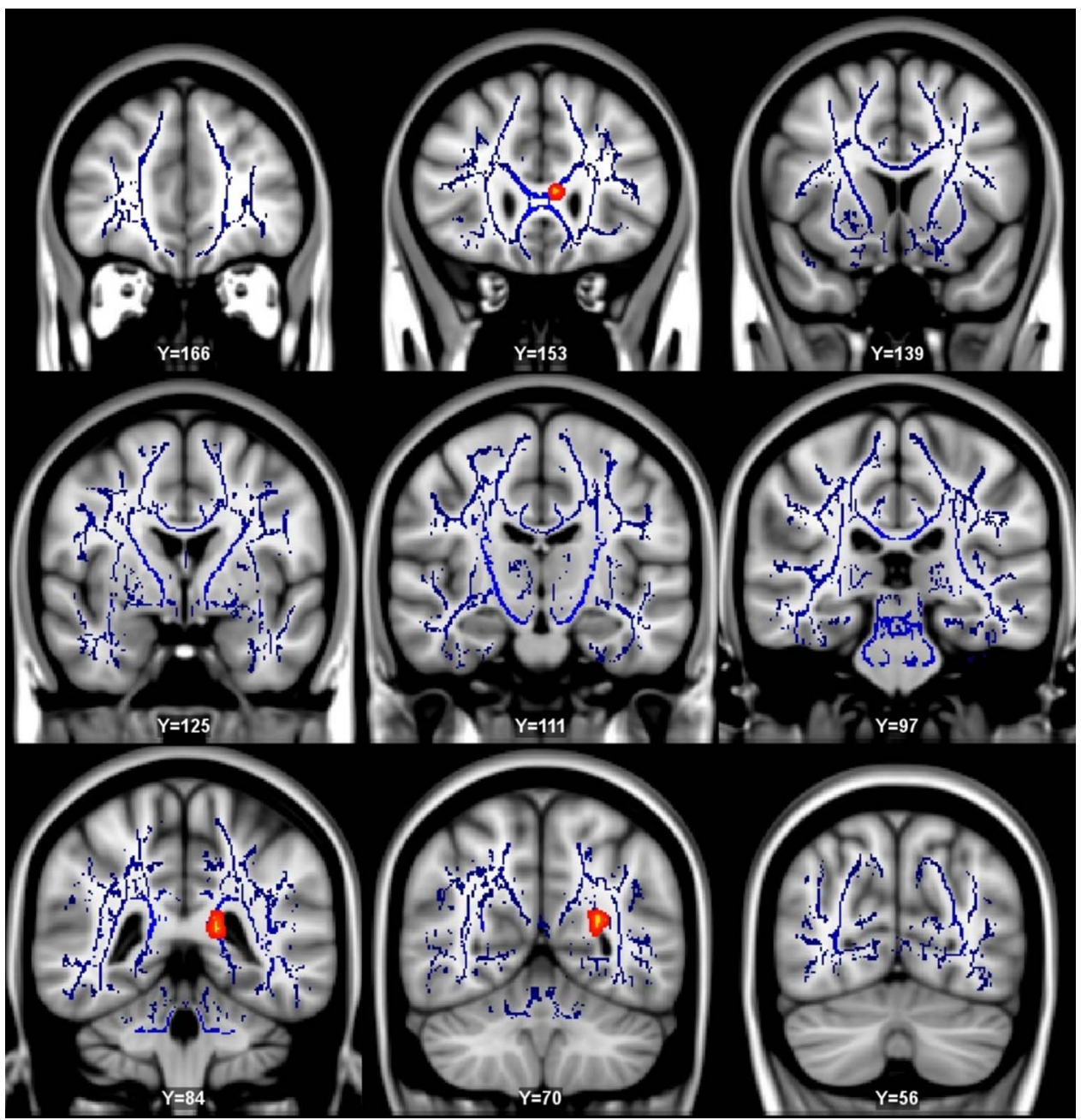

Figure 7 Significant voxel-wise correlations (red) between increased radial diffusivity (DR) and decreased total score on Rey Complex Figure Test in patients with Parkinson's disease superimposed on the mean fractional anisotropy map from all participants (blue). Significant correlations are mainly seen in posterior parts of corpus callosum. No voxels were significant for reduced DR. Multiple comparisons were corrected for by threshold-free cluster enhancement with the threshold set at $p<0.05$, and the significant voxels are inflated for ease of viewing. The statistical maps are shown as overlays on the Montreal Neurological Institutes template (annotated with the corresponding y-coordinates).

body disease suggest that DTI may be a useful biomarker for this type of neurodegeneration.

Limitations of our study include lack of postmortem diagnosis. However, in the case of predementia $\mathrm{AD}$, diagnosis was strengthened by use of CSF biomarkers and all patients with PD had a positive DaTSCAN supporting the clinical diagnosis.

The sample size is relatively low, and thus power issues make it difficult to reach statistical significance and differentiate between AD and PD-specific changes.

Not all patients with PD had measurable cognitive impairment. Thus, it is possible that more pronounced changes and stronger associations with cognition would have been detected if we had focused on patients with PD with established MCI.

Only FA and DR were evaluated, whereas other parameters, such as mean diffusivity, axial diffusivity, were not explored.
Author affiliations

${ }^{1}$ Department of Geriatric Psychiatry, Akershus University Hospital, Lørenskog, Norway

${ }^{2}$ University of Oslo, AHUS Campus, Oslo, Norway

${ }^{3}$ Department of Neurology, Akershus University Hospital, Lørenskog, Norway ${ }^{4}$ Department of Neurobiology, Alzheimer's Disease Research Centre, Care Sciences and Society, Karolinska Institutet, Stockholm, Sweden ${ }^{5}$ Centre for Age-Related Diseases, Stavanger University Hospital, Stavanger, Norway

${ }^{6}$ Department of Geriatric Psychiatry, Østfold Central Hospital, Fredrikstad, Norway

${ }^{7}$ The Intervention Centre, Oslo University Hospital, Rikshospitalet, Oslo, Norway

${ }^{8}$ Department of Physics, University of Oslo, Oslo, Norway

${ }^{9}$ Department of Psychology, University of Oslo, Norway

${ }^{10}$ Department of Radiology, Akershus University Hospital, Lørenskog, Norway

Contributors DA and TF planned and supervised the study. EA, VKK, PS and AH collected the data. EA and VKK performed the statistical analysis. EA wrote the first draft and all the authors critically reviewed and revised the manuscript. $A B$ was responsible for set-up and quality control of the MRI protocol. PS was responsible for processing of MRI data and voxel-wise 
statistical analyses. EH was responsible for the implementation of neuropsychological tests in cooperation with DA and TF. AE was responsible for coordination and interpretation of DaTSCAN.

Funding Southern and Eastern Norway Regional Health Authority (grant number 2011093) and Akershus University Hospital.

Competing interests DA has received honoraria or research support from Lundbeck, Inc, Novartis, GE Healthcare, and GlaxoSmithKline; serves on the editorial boards of International Psychogeriatrics, Movement Disorders, and the Journal of Neurology, Neurosurgery, and Psychiatry.

Patient consent Obtained.

Ethics approval South-Eastern Norway ethical committee for medical research.

Provenance and peer review Not commissioned; externally peer reviewed.

Data sharing statement No additional data are available.

Open Access This is an Open Access article distributed in accordance with the Creative Commons Attribution Non Commercial (CC BY-NC 3.0) license, which permits others to distribute, remix, adapt, build upon this work noncommercially, and license their derivative works on different terms, provided the original work is properly cited and the use is non-commercial. See: http:// creativecommons.org/licenses/by-nc/3.0/

\section{REFERENCES}

1. Svenningsson P, Westman E, Ballard C, et al. Cognitive impairment in patients with Parkinson's disease: diagnosis, biomarkers, and treatment. [Review]. Lancet Neurol 2012;11:697-707.

2. Bohnen NI, Albin RL. White matter lesions in Parkinson disease. [Review]. Nat Rev Neurosci 2011;7:229-36.

3. Kamagata K, Motoi Y, Abe O, et al. White matter alteration of the cingulum in Parkinson disease with and without dementia: evaluation by diffusion tensor tract-specific analysis. AJNR Am J Neuroradiol 2012;33:890-5.

4. Hattori T, Orimo S, Aoki S, et al. Cognitive status correlates with white matter alteration in Parkinson's disease. Hum Brain Mapp 2012;33:727-39.

5. Selnes P, Aarsland P, Bjørnerud A, et al. Diffusion tensor imaging surpasses cerebrospinal fluid as predictor of cognitive decline and medial lobe atrophy in subjective cognitive impairment and mild cognitive impairment. J Alzheimer Dis 2013;33:723-36.

6. Gelb DJ, Oliver E, Gilman S. Diagnostic criteria for Parkinson disease. [Review] [71 refs]. Arch Neurol 1999;56:33-9.

7. Dubois B, Feldman HH, Jacova C, et al. Research criteria for the diagnosis of Alzheimer's disease: revising the NINCDS-ADRDA criteria. [Review] [143 refs]. Lancet Neurol 2007;6:734-46.

8. Fjell AM, Walhovd KB, Amlien I, et al. Morphometric changes in the episodic memory network and tau pathologic features correlate with memory performance in patients with mild cognitive impairment. AJNR Am J Neuroradiol 2008;29:1183-9.

9. Sjogren $\mathrm{M}$, Vanderstichele $\mathrm{H}$, Agren $\mathrm{H}$, et al. Tau and $\mathrm{A}$ beta 42 in cerebrospinal fluid from healthy adults 21-93 years of age: establishment of reference values. Clin Chem 2001;47:1776-81.

10. Darcourt J, Booij J, Tatsch $\mathrm{K}$, et al. EANM procedure guidelines for brain neurotransmission SPECT using (123)|-labelled dopamine transporter ligands, version 2. Eur J Nucl Med Mol Imaging 2010;37:443-50.

11. Fahn S, Elton R. Members of the UPDRS development committee: Unified Parkinson's Disease Rating Scale. In Fahn S, Marsden CD, Calne DBGoldstein M, eds. Recent developments in Parkinson's disease. FlorhamPark: MacMillan Health Care Information, 1987:153-63.

12. Hoehn MH, Yahr MD. Parkinsonism: onset, progression and mortality. Neurology 1967;17:427-42.

13. Tomlinson CL, Stowe R, Patel S, et al. Systematic review of levodopa dose equivalency reporting in Parkinson's disease. [Review]. Mov Disord 2010;25:2649-53.

14. Almeida OP, Almeida SA. Short versions of the geriatric depression scale: a study of their validity for the diagnosis of a major depressive episode according to ICD-10 and DSM-IV. Int J Geriatr Psychiatry 1999;14:858-65.

15. Fazekas F, Chawluk JB, Alavi A, et al. MR signal abnormalities at $1.5 \mathrm{~T}$ in Alzheimer's dementia and normal aging. AJR Am J Roentgenol 1987;149:351-6.

16. Auer S, Reisberg B. The GDS/FAST staging system. Int Psychogeriatr 1997;9(1 Suppl):167-71.
17. Watson GS, Leverenz JB. Profile of cognitive impairment in Parkinson's disease. [Review] [58 refs]. Brain Pathol 2010;20:640-5.

18. Schmidt M. Rey auditory and verbal learning test. A handbook. Los Angeles, CA: Western Psychological Services, 1996.

19. Meyers Je, Meyers JR. Rey complex figure test and recognition trail. Lutz, FL: Psychological Assessment Resources, 1995.

20. Spreen O, Strauss EA. A compendium of neuropsychological tests, second edition: administrations, norms and commentary. New York: Oxford University Press, 1998.

21. Delis DC, Kaplan E, Kramer JH. Delis and Kaplan executive functions system: examiner's manual. San Antonio, TX: The Psychological Corporation, 2001.

22. Heaton RK, Miller SW, Taylor MJ, et al. Revised comprehensive norms for an expanded Halstead-Reitan battery: demographically adjusted neuropsychological norms for African American and Caucasian adults. Psychological Assessment Resources, 2004.

23. Boller F, Passafiume D, Keefe NC, et al. Visuospatial impairment in Parkinson's disease. Role of perceptual and motor factors. Arch Neurol 1984;41:485-90.

24. Pirozzolo FJ, Hansch EC, Mortimer JA, et al. Dementia in Parkinson disease: a neuropsychological analysis. Brain Cogn 1982;1:71-83.

25. Mosimann UP, Mather G, Wesnes KA, et al. Visual perception in Parkinson disease dementia and dementia with Lewy bodies. Neurology 2004;63:2091-6.

26. Watson GS, Leverenz JB. Profile of cognitive impairment in Parkinson's disease. [Review] [58 refs]. Brain Pathol 2010; 20:640-5.

27. Abe Y, Kachi T, Kato T, et al. Occipital hypoperfusion in Parkinson's disease without dementia: correlation to impaired cortical visual processing. J Neurol Neurosurg Psychiatry 2003;74:419-22.

28. Colloby SJ, Fenwick JD, Williams ED, et al. A comparison of (99 m) Tc-HMPAO SPET changes in dementia with Lewy bodies and Alzheimer's disease using statistical parametric mapping. Eur J Nucl Med Mol Imaging 2002;29:615-22.

29. Ungerleider LG, Haxby JV. 'What' and 'where' in the human brain. [Review] [86 refs]. Curr Opin Neurobiol 1994:4:157-65.

30. Diederich NJ, Goetz CG, Stebbins GT. Repeated visual hallucinations in Parkinson's disease as disturbed external/internal perceptions: focused review and a new integrative model. [Review] [93 refs]. Mov Disord 2005;20:130-40.

31. Alvarez JA, Emory E. Executive function and the frontal lobes: a meta-analytic review. [Review] [199 refs]. Neuropsychol Rev 2006;16:17-42.

32. Cummings JL. Frontal-subcortical circuits and human behavior. [Review] [71 refs]. Arch Neurol 1993;50:873-80.

33. Woolrich MW, Jbabdi S, Patenaude B, et al. Bayesian analysis of neuroimaging data in FSL. Neuroimage 2009;45(1 Suppl): S173-86.

34. Smith SM, Jenkinson M, Johansen-Berg $\mathrm{H}$, et al. Tract-based spatial statistics: voxelwise analysis of multi-subject diffusion data. Neuroimage 2006;31:1487-505.

35. Rueckert D, Sonoda LI, Hayes C, et al. Nonrigid registration using free-form deformations: application to breast MR images. IEEE Trans Med Imaging 1999;18:712-21.

36. Fischl B, Salat DH, Busa E, et al. Whole brain segmentation: automated labeling of neuroanatomical structures in the human brain. Neuron 2002;33:341-55.

37. Fischl B, van der Kouwe A, Destrieux C, et al. Automatically parcellating the human cerebral cortex. Cereb Cortex 2004:14:11-22.

38. Desikan RS, Segonne F, Fischl B, et al. An automated labeling system for subdividing the human cerebral cortex on MRI scans into gyral based regions of interest. Neuroimage 2006: 31:968-80.

39. Zhan W, Kang GA, Glass GA, et al. Regional alterations of brain microstructure in Parkinson's disease using diffusion tensor imaging. Mov Disord 2012;27:90-7.

40. Watson R, Blamire AM, Colloby SJ, et al. Characterizing dementia with Lewy bodies by means of diffusion tensor imaging. Neurology 2012;79:906-14.

41. Kamagata K, Motoi $\mathrm{Y}$, Tomiyama $\mathrm{H}$, et al. Relationship between cognitive impairment and white-matter alteration in Parkinson's disease with dementia: tract-based spatial statistics and tract-specific analysis. Eur Radiol 2013;23:1946-55.

42. Meltzer TR, Watts R, MacAskill MR, et al. White matter microstructure deteriorates across cognitive stages in Parkinson disease. Neurology 2013;80:1841-9.

43. Agosta F, Canu E, Stefanova E, et al. Mild cognitive impairment in Parkinson's disease is associated with a distributed pattern of brain white matter damage. Hum Brain Mapp 2013. doi: 10.1002/hbm. 22302. [Epub ahead of print]. 